\title{
Addressing Catalytic Material Problems using Automated Feature Analysis
} David Pollick $^{1}$ and Patricia Nelson ${ }^{1}$

\author{
${ }^{1}$ BASF Corporation, Catalyst Division, Iselin, NJ, USA
}

Electron microscopy and microanalysis have always been indispensable tools for characterizing material properties, which are strongly correlated to the overall, catalytic performance of a finished commercial product. Historically, most of the feature analysis relies heavily upon visual judgment and rigorous measurements of an electron micrograph by the microscope operator. However, such processes suffer from inconsistencies due to human errors, especially when analysis was performed by different operators. Furthermore, the rigorous measurements are time-consuming and provide poor statistical results. In a commercial setting, where analysis throughput, consistency and efficiency are of essence, a more robust approach is needed. Automated image analysis provides a quantitative means for objectively differentiating among similar materials with improved speed, essential statistics and consistency.

In this paper, we describe feature analysis performed on catalytic materials using a software suite provided by Bruker-AXS within their Quantax ESPRIT imaging and spectroscopy system [1]. High resolution scanning electron microscopy (SEM) images were first acquired, followed by image-filtering and grey-level 'thresholding' to enhance and/or isolate specific attribute(s) of interest. Specifically, the software provides flexibility for establishing either a binary or multiphase histogram for control and fine tuning of phase separations. Automation-related errors can be further minimized through selection rules. For example, features at the image borders can be excluded; image noise and fine contaminants can be filtered out by ignoring features smaller than a user-defined critical size. Once the feature analysis 'macro' is defined, the software allows for 30 physical properties such as, volume, phase, roughness, length etc. to be rapidly determined. The feature analysis routine can be saved and reused for consistency. Results derived from each micrograph can be appended for larger sampling and better statistics.

Combining energy-dispersive X-ray spectroscopy (EDS) with automated feature analysis transforms the technique into a powerful tool for solving technical problems by adding another dimension to the characterization. Chemical composition, phase analysis and physical attributes can be correlated, again, through automated algorithms. The synergistic approach allows one to find answers to questions like: 'how many $30 \mu \mathrm{m}$ particles have $3 \%$ alumina?' or 'what is the average diameter of particles with less than $85 \%$ silica?'

Through automated image and phase analysis, rapid characterization of physical attributes and chemistry to gain insights in the performance of commercial catalyst materials is made possible. The flexibility of the software allows screening of complex materials to be achievable. Results can be accurate, consistent and independent, which is important in a high-throughput environment.

[1] Bruker-Axis Quantax, User Manual, (2011) 

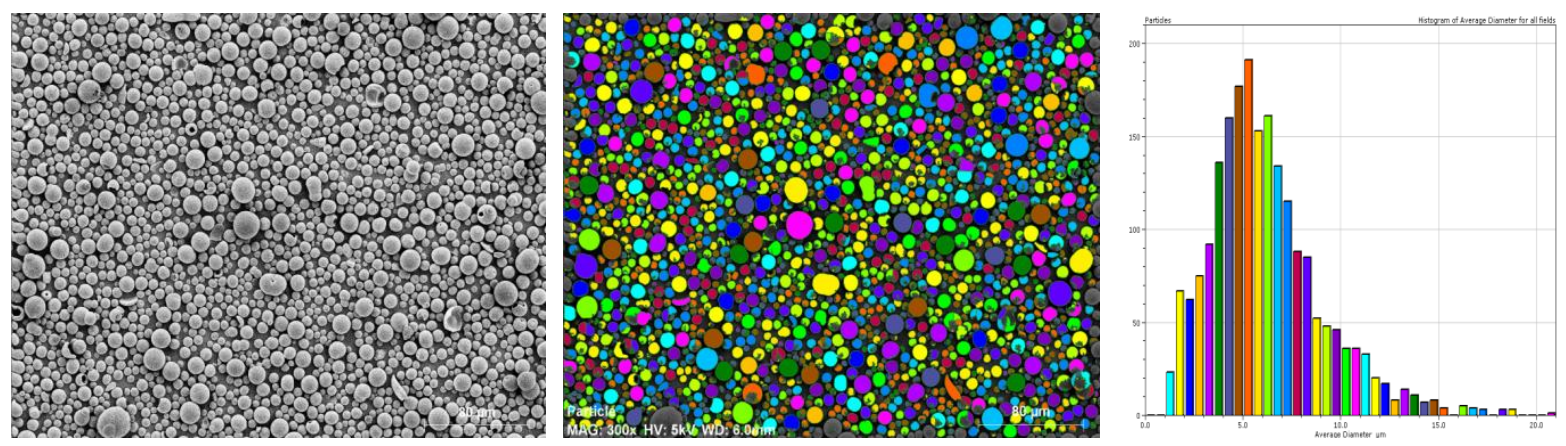

\begin{tabular}{|l|c|c|c|c|c|c|}
\hline Particle & Area & Average Diameter & Aspect Ratio & Shape Factor & Circularity & Roughness \\
\hline Avg & 30.9 & 6.16 & 1.36 & 0.62 & 1.69 & 1.16 \\
\hline StdDev & 34.5 & 2.90 & 0.35 & 0.21 & 0.63 & 0.17 \\
\hline Min & 0.8 & 1.25 & 1.02 & 0.12 & 1.06 & 0.95 \\
\hline Max & 321.4 & 20.53 & 6.57 & 0.99 & 7.56 & 2.15 \\
\hline
\end{tabular}

Figure 1. SE Image, Processed Image, Binned Histogram, and Resulting Table of Powdered Catalyst
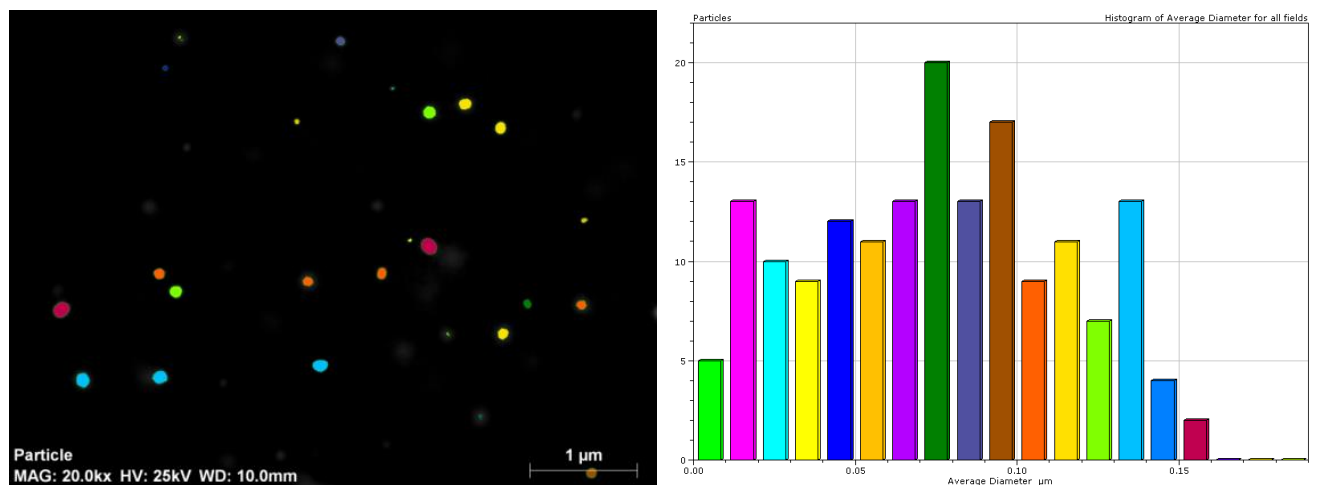

Figure 2. Variation in Precious Metal Average Diameter after Aging Protocol 